COGNITIVE SCIENCE 8, 255-273 (1984)

\title{
The Role of a Mental Model in Learning to Operate a Device*
}

\author{
DAVID E. KIERAS AND SUSAN BOVAIR \\ University of Michigan
}

\begin{abstract}
This report presents three studies concerned with learning how to operate a simple control panel device, and how this learning is affected by understanding a device model that describes the internal mechanism of the device. The first experiment compared two groups, one of which learned a set of operating procedures for the device by rote, and the other learned the device model before receiving the identical procedure training. The model group learned the procedures faster, retained them more accurately, executed them foster, and simplified inefficient procedures far more often, than the rote group. The second study demonstrated that the model group is able to infer the procedures much more easily than the rote group, which would lead to more rapid learning and better recall performance. The third study showed that the important content of the device model was the specific configuration of components and controls, and not the motivational aspects, component descriptions, or general principles. This specific information is what is logically required to infer the procedures. Thus, the benefits of having o device model depend on whether it supports direct and simple inference of the exact steps required to operate the device.
\end{abstract}

This paper is concerned with the role of a mental model in learning how to operate an unfamiliar piece of equipment. By "mental model" is meant some kind of understanding of how the device works in terms of its internal structure and processes (cf. Halasz \& Moran, 1983; Norman, 1983). In the remainder of this paper, this type of mental model will be termed a device model, to distinguish it from the many other senses of the term mental model, such as that used by Johnson-Laird (1980).

*This research was supported by the Personnel and Training Research Programs, Office of Naval Research, under Contract Number N00014-81-C-0699, Contract Authority Identification Number NR157-473. Request for reprints or further information should be sent to David Kieras, Program in Technical Communication, College of Engineering, University of Michigan, Ann Arbor, MI 48109. 
Much of the recent discussion of mental models (see Gentner \& Stevens, 1983) has been mainly concerned with extremely complex physical systems, or with behavior in relatively ill-defined tasks, such as describing how an electrical circuit works. The emphasis has been on mental models in which the understanding of the system approaches the level of understanding that an actual expert in the relevant technical field would have. For example, considerable attention has been devoted to how people acquire a fundamental understanding of electrical circuits (Gentner \& Gentner, 1983) or of a complex shipboard steam propulsion system (Williams, Hollan, \& Stevens, 1983). However, there have been few empirical studies of mental model effects, and many of the results are unclear. Likewise, there has been very little study of the role of mental models in interacting with relatively simple devices. This domain is not only of great practical importance, but also is well suited to experimentation and modelling.

There are two very strong intuitions about the role of a device model in learning how to operate a device, or in being able to operate it once it is learned. One, held by most psychologists, is that having such knowledge would be of great value; the other intuition, held by the technological industries, is that device models are in fact unnecessary (see Bond \& Towne, 1979; Kieras \& Polson, 1982, in press), for more discussion). For example, the modern telephone system, with its many special options, is extremely complex, but the typical telephone book contains only "how-to-do-it" instructions; very few people know how the system works beyond the crudest principles. However, almost everybody can successfully operate a telephone. Detailed knowledge of how the system works seems to be irrelevant. Another example is the instructional material that accompanies word processing systems. Some major manufacturers of such systems have apparently adopted the policy that instructional material should focus just on "how to get the job done," and should not contain any how-it-works knowledge. This judgement is not badly wrong, because many people learn how to oeprate these system successfully within a reasonable amount of time.

For phychologists, the great value of having a device model is intuitively compelling. There are classic results in which a procedure was remembered better if subjects were taught the underlying principle (e.g., Katona, 1940; Wertheimer, 195.9). However, recent experimental attempts to demonstrate positive effects of teaching subjects a device model for a computer system have been inconclusive. For example, in learning a text editor, nonsignificant results of device model training were observed by Foss, Smith, and Rosson (1982) and Alexander (1982). Halasz and Moran (1983), using an ordinary calculator, discovered strong positive effects on having a device model in some tasks, but not others. Since many of these are null results, they have not been published in the archival literature; there are probably many more failures to demonstrate such effects. Thus, the role of 
device models in operating equipment remains very unclear, despite these strong intuitions and the importance of the practical questions involved.

This paper reports three studies. The first is a demonstration that providing a device model can result in faster learning and better retention of operating procedures. The second demonstrates that the device model can be used to infer the operating procedures. The third examines what is the critical type of information in the device model materials. For brevity, the methods and results have been substantially condensed; for more detail on Experiments 1 and 2, refer to Kieras and Bovair (1983), and Kieras (1984) for Experiment 3.

\section{EXPERIMENT 1}

Two groups of subjects learned a set of procedures for operating a simple control panel device consisting of switches, pushbuttons, and indicator lights. The goal of the procedures was to get a certain indicator light to flash. The device model group learned some how-it-works knowledge in the form of a description of the device based on the familiar television science fiction series Star Trek. Namely, they were taught that the device was the control panel for a "phaser bank" on the "Starship Enterprise," with the flashing light indicating a successful firing of the phaser bank. The internal components and processes of the device were explained in terms of this fictitious system. To ensure that the device model manipulation was effective, subjects not only studied the model materials, but also had to successfully pass a test for knowledge of the model before proceeding to the procedure training. The device model materials contained no description of operating procedures.

The rote group received no model training, but only learned the procedures "by rote." The two groups received exactly the same procedure training; this was made possible by referring to the device controls with abbreviations that could be used for both groups. Since a device model should enable subjects to operate a device more efficiently, some of the procedures were made deliberately inefficient, and the subjects were given the opportunity to devise more efficient procedures. After learning the procedures, both groups were tested immediately and after one week for retention of the procedures.

\section{Method}

The device and device model. The device consisted of a control panel with a toggle switch, a three-position rotary selector switch, two push buttons, and four indicator lights. A sketch of the control panel appears in Figure 1. The 
user had no direct knowledge of the internal state or organization of the device; the only aspects of the device that the user was directly aware of was the settings of the switches, and whether the indicator lights were on, off, or flashing. The simplest way to explain the behavior of the device is to present the actual device model that was presented to the subjects.

Figure 2 is the diagram that was shown to the subjects who learned the device model. The toggle switch labelled $S P$ (shipboard power) is the on/off switch. If the $S P$ switch is on, the $S P$ indicator lights. The power flows into an energy booster $(E B)$, which, if operating correctly and receiving power, lights the $E B$ indicator. Power flows out of the energy booster into two accuumulators, labelled $M A$ (main accumulator) and $S A$ (secondary accumulator). The main accumulator has an indicator which shows that the accumulator is receiving energy and functioning properly. The secondary accumulator has no such indicator, and its condition is not indicated to the user. The rotary switch ESS (energy source selector) selects which accumulator is to be connected to the $P B$ (phaser bank). If the corresponding pushbutton is pressed, energy flows from the accumulator to the phaser bank. When the phaser bank receives the energy, the indicator labelled PFI (phaser firing indicator) flashes 4 times at roughly half second intervals, and then stops.

The subjects studied written material along with the diagram. To provide an impression of these materials, a sample excerpt appears in Table I; the complete materials are in Kieras and Bovair (1983). The key features of the device model materials were as follows: It was based on a simple description of the major components in Figure 2, along with a description of how these components related to each other, and how the controls controlled the flow of "energy" from one component to another. The training had a small amount of discussion of how each of the components worked, mainly emphasizing that they sometimes malfunctioned. It is important to note that the materials did not contain any description of procedures to be followed. After studying the device model, subjects were given a multiple choice test on the model. If they missed any questions, they were required to study the material again until all questions were answered correctly.

The operating procedures. The subjects learned two kinds of procedures for operating the device, normal and malfunction procedures. The two normal procedures for operating the device, which were designated simply as Procedure $l$ and Procedure 2, correspond to the two different accumulators. These procedures consisted of the following steps: turn $S P$ switch on; set $E S$ selector to $M A$ (or $S A$ ); press button $F M$ (or $F S$ ); wait until $P F$ indicator finishes flashing; set $E S$ selector to $N$; turn $S P$ off. After learning these two normal procedures, the subjects were then told that sometimes the device malfunctioned, and depending on the situation, it could be made to work sometimes by following an alternative procedure. These malfunction 


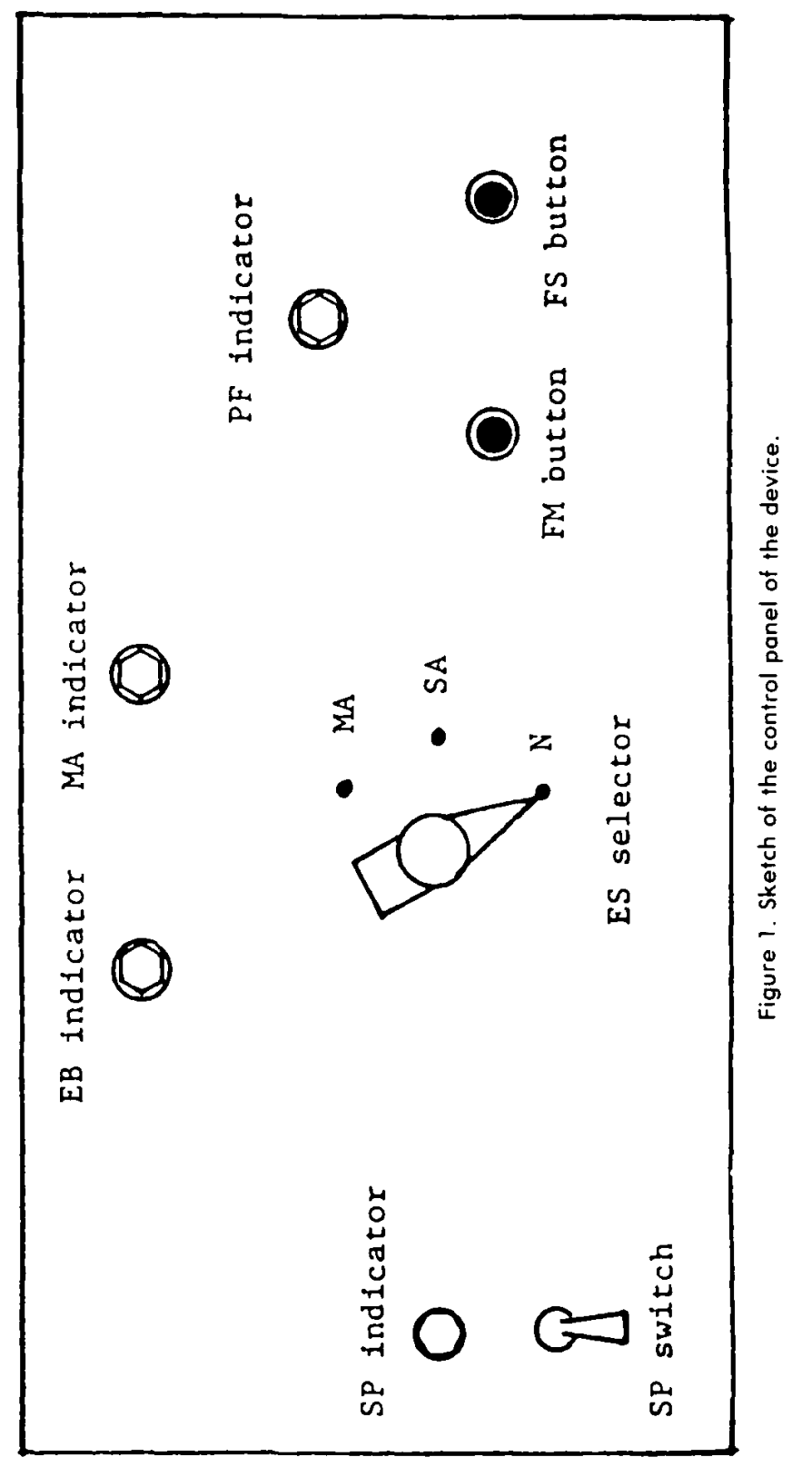




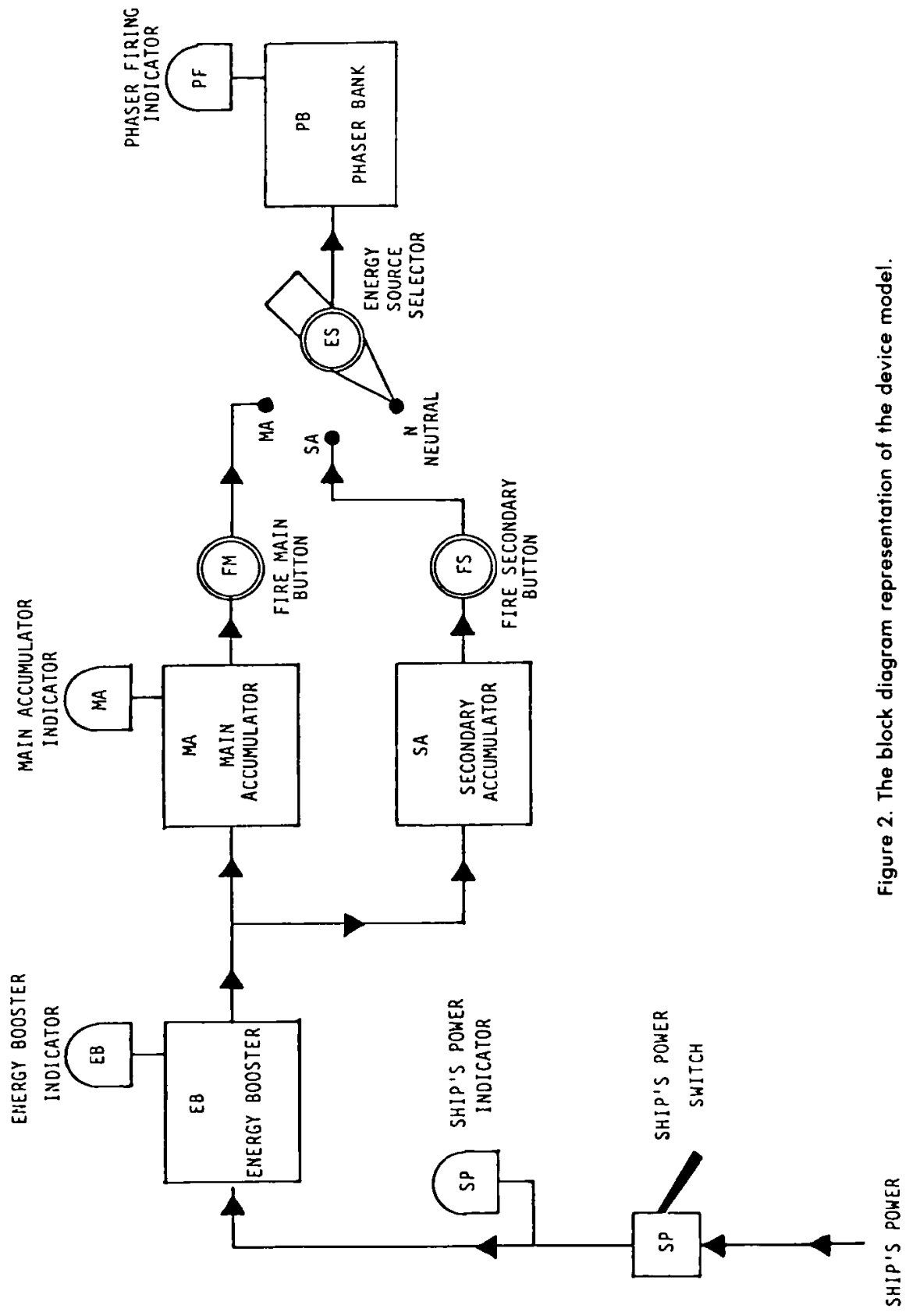


TABLE I

Sample of the Device Model Instructions for Experiment 1

The energy booster takes in power from the ship and boosts it to the level necessary to fire the phasers. Power that has been boosted by the energy booster is fed into the two accumulators. Both accumulators store lorge amounts of power ready to be discharged to the phaser bank whenever the phasers are fired.

Because the accumulators handle such large amounts of power, if they are used continuously they are liable to overload and burn out. To prevent continuous use of one accumula10r, this system has two: the main accumulator (MA) and the secondary accumulator (SA).

The power coming in from the shipboard circuits is controlled by the ship's power switch (SP). When this switch is off, no power is being drown from the ship. When the switch is furned on, power is drawn from the ship into the energy booster. The boosted power is then fed into the accumulators. The accumulator whose energy will be discharged to the phaser banks is selected by the energy source selector (ES). While the ES selector is set to neutral $(N)$, no energy can be discharged from either accumulator to the phaser bank.

situations were of two types; in one type, the PF indicator could be made to flash by means of an alternate procedure, such as using the $S A$ setting instead of the $M A$. In the other type, the PF indicator could not be made to flash, and the subject was to follow a procedure to indicate that an irrecoverable malfunction has occurred. This consisted of typing the letter " $E$ " on the terminal keyboard, and then "shutting down" by setting the $E S$ selector to $N$ and turning the $S P$ switch off. For each normal procedure, four different malfunction situations were defined. This gave a total of 10 procedures for the subject to learn. The last step in each procedure consisted of typing the letter " $F$ " for "finished."

Four of the procedures were designed to be inefficient. In these procedures, the subject was trained to follow the complete normal procedure up to the point where the $P F$ indicator was supposed to flash. However, it was possible to tell from the indicators, as soon as the power was turned on, whether the desired procedure would work at all, and steps could then be immediately taken to either try the other accumulator, or to simply shut down the system. By including such procedures, it was possible to determine whether subjects would be able to simplify, or "short-cut," the instructed procedures.

Apparatus. The device itself was simulated by means of a laboratory computer which monitored the settings of the switches and push buttons and controlled the indicator lights accordingly. All instructions and commands to the subjects were presented on a standard video terminal positioned next to the device. A computer-assisted instruction program was used to present the device model training, the procedure training, and the tests for retention of procedures. The subject was seated in a small room at at table with the terminal and the control panel, and was observed by means of closed-circuit television. 
Procedure. The device model group and the rote group received exactly the same procedure training. Both groups were familiarized with the location and labels of the controls at the beginning of the experiment. The only difference between the two groups were that the device model group received the device model instruction at the beginning of the experiment, and a small amount of additional device model instruction, before starting to learn the malfunction procedures, and had the diagram (Figure 2) posted on the wall in front of them throughout training, but not during retention testing.

The process of training each procedure was a serial anticipation teaching phase alternating with a test phase. After performing the last step in the teaching phase, the subject entered the test phase and attempted to execute the procedure from memory. If the entire procedure was performed correctly, another test trial was begun. If three correct trials in a row were performed, the subject then moved on to the next procedure. If an incorrect step was made, the subject was immediately informed, and then began the teaching phase again.

After learning all 10 procedures, the subject performed three retention tests. In each test, a command to perform either Procedure 1 or Procedure 2 would appear on the subject's terminal, and he or she would then attempt to perform the designated procedure. On half of the 16 trials, a normal situation was the case, so the normal procedure would work; on the other half, there was some malfunction situation, and the subject had to perform the appropriate malfunction procedure. No feedback was given during the tests. The data recorded were which control was operated on each step of the subject's procedure, and the corresponding response latency. Test 1 was given immediately after completing the procedure learning, with subjects being instructed to perform the procedures exactly as they had learned them. After completing the first test, the subject performed Test 2, which was identical to the first test, but they were instructed to try to short-cut or simplify the procedures if possible. Following the second test, the subject was sent home, with instructions to return in one week for Test 3 , which was the same test, but with no particular instructions given with regard to shortcuts.

Subjects. The 40 subjects, 20 in each group, were recruited through campus advertisements and they were paid $\$ 5$ for each session. Subjects were randomly assigned to either model or rote group so that there were always approximately equal numbers of subjects in each group. Subjects were balanced by sex so that there were an equal number of males and females in each group. Three of the subjects did not return for their second session, resulting in 19 subjects in the rote condition and 18 in the model condition. A one week delay was intended, but, due to scheduling problems, two subjects returned ten days after the first session, while 4 returned eight days later. 


\section{Results}

For reasons of brevity, differences between procedures will be ignored. While there are many detailed procedure-specific aspects of the results, the major thrust of the results is not affected by ignoring the procedures factor.

Table II presents a summary of the effects of the device model manipulation. All effects reported here are statistically significant at or beyond the .05 level, using appropriate tests. Subjects in the model group took an average of $1141 \mathrm{~s}$ to learn the device model; this time included reading the training material and performing the quiz to the criterion of all questions answered correctly. Subjects in the model group learned a procedure faster than the rote subjects, by an average of $76 \mathrm{~s}$, a $28 \%$ improvement. The retention test performance was higher for the model group. Note that the model group executed a correct (in terms of achieving the final goal) procedure $19 \%$ more often over all three tests; however, this improvement was a modest $11 \%$ for the 1-week delayed test considered separately. Especially noteworthy is the strong difference in the number of short-cut procedures used when it was possible. The model group uses these efficient procedures far more often than the rote group. Finally, the model group executed the procedures $17 \%$ faster during the tests than the rote group, even when, as shown in the table, only the exact reproduction of the instructed procedures is considered.

TABLE ॥

Summary of Results on Learning Procedures With and Without a Device Model

\begin{tabular}{lccc}
\hline & \multicolumn{3}{c}{ Group } \\
\cline { 2 - 4 } & Rote & Model Improvement \\
\hline Mean Device Model Training Time (s) & 1141 & $\ldots$ \\
Mean Procedure Training Time (s) & 210 & 194 & $28 \%$ \\
Mean Correct Procedure Retention (over all tests) & $67 \%$ & $80 \%$ & $19 \%$ \\
Mean Correct Retention After 1 Week & $71 \%$ & $78 \%$ & $11 \%$ \\
Proportion of "short-cuts" (more efficient & $8 \%$ & $40 \%$ & $400 \%$ \\
procedures done when possible) & & & $17 \%$ \\
Mean execution time of retained instructed & & & \\
procedures (s) & 20.1 & 16.8 & \\
\hline
\end{tabular}

\section{Discussion}

Note that no attempt was made to optimize the device model materials. Nonetheless, over the entire procedure training on the 10 procedures, model subjects were an average of about $760 \mathrm{~s}$ faster than rote subjects, which compares favorably with the average $1141 \mathrm{~s}$ that it took subjects to learn the device model. Thus, in roughly similar total training times, the model group 
remembered instructed procedures more accurately, used more efficient procedures much more often, and executed the procedures faster than the rote group.

This experiment shows that having a device model does improve performance on learning and retaining the operating procedures for a device. This result is significant, because it has been hard to demonstrate that such effects do exist. However, the question is how knowledge of the device model produces these effects. They are similar to those which have been obtained in a variety of learning situations in which the more "meaningful" the material is, the faster it is learned and the better it is retained. However, to say that the device model makes the device more "meaningful" does not actually explain how this extra descriptive information makes the procedural information easier to learn and remember.

The specific hypothesis offered here is that knowledge of how a system works helps by enabling the user to infer how to operate the device. It is an old and established principle of learning that learning and memory will be facilitated if the learner can reconstruct the to-be-remembered information. Perhaps the device model simply allows the model group subjects to reconstruct by means of inference the operating procedures even if specific details of the direct memory of them have been forgotten. This provides a specific explanation for how making a device "meaningful" with a device model can allow the procedures to be learned and remembered better.

\section{EXPERIMENT 2}

An obvious implication of the above hypothesis is that, if subjects are asked to infer procedures, rather than learn them, the model group subjects should have a decided advantage. Experiment 2 tested this implication by using two groups as before, in which one learned the device model and the other did not, but they were asked to infer the procedures while "thinking out loud." It was expected that the subjects with the device model would be able to infer the procedures quite readily, and that the think-aloud protocol data would show that they were basing their inferences on the device model. In contrast, the rote group would be forced to rely on very general aspects of how one operates equipment, such as the fact that it has to be turned on, and would follow some kind of trial and error approach to discovering the opening procedures.

\section{Method}

Subjects. Subjects were sampled from the same pool as in Experiment 1, and were paid $\$ 5$. Subjects were run individually, being assigned to their 
conditions at random. Since this was a protocol-collection experiment, only five subjects wre run in each group.

Instructions and procedure. The equipment and device model training were the same as for Experiment 1. The instructions for the procedure inference task told rote subjects that the goal of operating the device was to make the $P F$ indicator flash, and that they should discover the control settings that make this happen. They were asked to "think aloud" about their guesses, hypotheses, and the knowledge they were using. After they had found settings that would make the $P F$ indicator flash, they were asked to develop a procedure with as few steps as possible, and then go on to find a second way to make the $P F$ indicator flash. Instructions to the model subjects were similar, except that they were told that the goal was to make the phasers fire. The model subjects had the device diagram displayed about the device throughout the whole experiment.

After subjects had read the instructions on the terminal, the procedure inference task was begun, with all statements and activities of the subject recorded on videotape; the lab computer recorded each change of the controls on the device. The experimenter prompted the subjects with questions if necessary to encourage "thinking out loud." The subjects inferred the procedures in two phases; the first considered of inferring the two normal situation procedures; the second phase consisted of inferring the malfunction situation procedures. Subjects were given two or three passes through all situations, in order to allow them to arrive at a stable procedure for each situation. The experimenter judged whether the subject's procedure was stable on the basis of correct performance, consistent control settings, and lack of hesitation.

\section{Results}

The basic measure of how easy it was to infer a procedure is the number of actions, defined as a change in control settings, tried before arriving at the appropriate goal state. The number of actions tried averaged over all 10 procedures for each attempt is shown in Table III. Both groups ended up with the same optimal procedures, but the rote group tried a very large number of actions in their first attempt. In contrast, almost all of the model subjects deduced the correct procedures almost completely correctly on their first attempt. These effects are significant at or beyond the .05 level.

The protocol data will be described very briefly. There was essentially no overlap in the protocol content between the two groups. The model group explained their actions and the device behavior almost completely in terms of the model. The rote group tended to follow a systematic trial and error strategy, and explained the device in terms of superficial relations be- 
TABLE III

Mean Number of Actions Tried While Inferring Procedures

\begin{tabular}{lrcc}
\hline & \multicolumn{3}{c}{ Attempt } \\
\cline { 2 - 4 } Group & 1 & 2 & 3 \\
\hline Rote & 21.8 & 9.8 & 8.1 \\
Model & 8.6 & 7.9 & $\ldots$ \\
\hline
\end{tabular}

tween the indicators or control labels. Especially interesting is that the rote subjects made many more statements that expressed negative affect or that the experiment was "rigged" in some way.

\section{Discussion}

Having the device model available while inferring the procedures for operating the device produced very powerful differences in the performance of the subjects and in the protocol data. The group with the device model inferred the procedures for operating the device in almost the fewest trial actions possible. As reported in detail in Kieras and Bovair (1983), they also engaged in very few "nonsense" trial actions, making only attempts that were consistent with the device model. One of the few cases where this was not so is one model group subject who made a couple of extraneous actions while discovering the first procedure. This subject tried to fire the phasers without first powering up the system, saying as he did so that he wanted to see if the accumulators still had a stored charge that could be used to fire the phaser. Thus, even when a model subject did not perform optimally, he did so on the basis of reasonable inferences from the device model. Clearly, the device model provided a basis for inferring the procedures.

\section{EXPERIMENT 3}

The results of Experiment 2 suggest very strongly that the facilitation observed in Experiment 1 was due to the model subjects being able to infer the procedures. However, the device model material contained many different types of information. It placed the device in an interesting and familiar fantasy context, which may have been more motivating than the rote condition. It also provided some general principles and design rationale information, such as why an energy booster is necessary. Finally, the device model contained information on the system topology (what is connected to what), and made use of the principle of power flow, in which power starts at a source and is routed through controls and components to a destination. 
An analysis in the form of a computer stimulation (described in Kieras, 1984) suggests that it is the system topology together with the power flow concept that makes inference of the procedures possible. But this is true only if the topology information describes specifically which controls are on what power-flow paths. Thus, the critical how-it-works information is the specific descriptions of the controls and their path relations to the internal components. Therefore, neither the fantasy context, nor details about the nature of the components, nor general principles about how the system works, should be of value in enabling subjects to infer the procedures. This set of assertions was tested in Experiment 3, which was also designed to collect detailed inter-response times during procedure inference. This time data is discussed in Kieras (1984).

\section{Method}

Materials and Design. The experiment was a $2 \times 2$ factorial design, with the factors being the presence or absence of the fantasy context and the presence or absence of specific control information. The no-fantasy no-specific condition was identical to the previous rote condition, and the fantasy specific condition was essentially the same as the previous model condition. A sample excerpt of the fantasy-specific materials is shown in Table IV. The changes from Table I materials are concerned only with ensuring that subjects refer to the diagram (a problem in Experiments 1 and 2) and changes in abbreviations to accomodate the other conditions more easily. The same diagram was used as in Experiment 1, with changes only in the abbreviations for the controls.

The no-fantasy specific condition subjects studied device model materials identical to the previous materials, except that all references to the Star Trek fantasy were eliminated, along with any discussion of how the system components worked or why they were present. The names of the components were changed to terms that did not convey any particular function for the system, such as pulser instead of phaser bank. Table $\mathrm{V}$ contains a sample of these materials that corresponds to Table IV. These subjects studied the same diagram as the fantasy-specific group, with appropriate changes in the labels.

The fantasy no-specific materials consisted of a fantasy explanation of the pseudo-physics principles underlying the phaser system, along with the major components and general power flow, but without describing any of the controls, indicators, or actual power-flow paths. This material was similar in length to the other device model materials, and was also accompanied by a diagram. The diagram is shown in Figure 3 , and a sample of the materials in Table VI. As in the other device model conditions, subjects had 
TABLE IV

Somple of Materials for the Fantosy Specific Condition

The arrows on the diagram show how power flows through the system. Starting on the lower left of the diagram, you can see that power comes in from the shipboard circuits. Notice on the diogram that this power flows to the energy Booster (B), and from there it flows to the fwo accumulators (MA and SA). The diagram shows that power can flow from either of the accumulators to the Phoser bank $(P)$. The switch, selector and pushbuttons control the flow of power.

I will first describe the function of each component, and then will describe how the controls relate to the components.

Ship's power cannot be used to fire the phaser directly becouse it is not at a high enough level. The energy Booster boosts the ship's power to the high level necessary to fire the phaser. Both accumulators store large amounts of power, and if they are used continuously. they are liable to overload and burn out. To prevent continuous use of one accumulator, this system has two: the Main Accumulator (MA) and the Secondary Accumulator (SA). When the Phaser bank receives power, rapid phase shifts take place. These phase shifts cause the emission of the phaser beams, and thus the actual firing.

Now that you have seen what each component does, I will describe how the controls relate to the operation of the components.

On the lower lett of the diagram, locate the ship's Power Switch (PS). You can see that the power coming in from the shipboard circuits is controlled by the PS switch. When this switch is off, no power can come in. When the switch is turned on, power flows into the energy Booster (B). Power from the energy Booster then flows into both accumulators. Find the selector on the diagram and notice that the accumulator whose power will be supplied to the Phoser bonk is selected by the selector $(S)$. While the selector is set to neutral $(N)$, no power can flow from either accumulator to the Phaser bank. When the selector is set to MA, the power can flow from the Main Accumulator. When the selector is set to SA, then power can flow from the Secondary Accumulator.

TABLE V

Sample of Materials for the Specific No-Fantasy Condition

The arrows on the diagram show how power flows through the system. Starting on the lower left of the diagram, you can see that power comes in from the power source. Notice on the diagram how this power flows to the Butfer $(B)$, and from there it flows to the two activators (MA and SA). The diagram shows that power can flow from either of the activators to the Pulser (P). The switch, selector, and pushbuttons control the flow of power.

I will now describe how the controls relate to the components.

On the lower left of the diagram, locate the power switch (PS). You can see that the power coming in from the power source is controlled by the PS switch. When this switch is off, no power can come in. When the switch is turned on, power flows into the Buffer (B). Power from the 8uffer then flows into both activators. Find the selector on the diagram, and notice that the activator whose power will be supplied to the Pulser $(P)$ is selected by the selector $(S)$. While the $S$ selector is set to neutral $(N)$, no power can flow from either activator to the Pulser (P). When the selector is set to MA, the power can flow from the Main Activator. When the selector is set to SA, then power can flow from the Secondary Activator. 

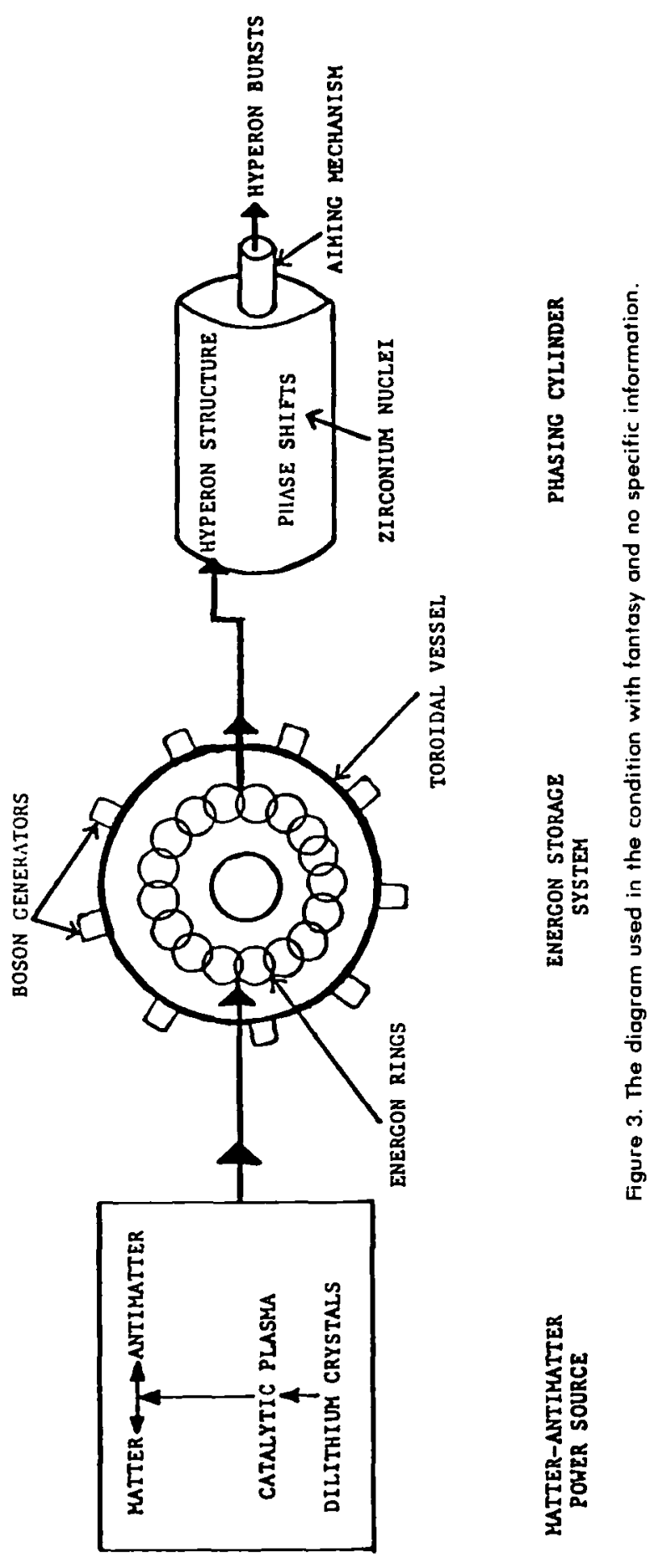
to pass a test on the content before proceeding to the rest of the experiment. Complete copies of the materials appear in Kieras (1984).

Subjects. Subjects were recruited and paid as in the previous experiments. Since it was desired to collect timing data for the two specific information groups, 10 subjects were run in these conditions, and 5 in the other two.

Procedures. This experiment used a simpler procedure than Experiment 2. Subjects made only one attempt to infer how to operate the device in each situation, and the normal and malfunction situations were not distinguished to the subject. All 10 situations were given to each subject. After completing each situation, the subject was prompted for a retrospective report; however, these data will not be reported.

TABLE VI

Sample of Materials for the Fantasy with no Specific Information Condition

The phaser system is based on several important principles in physics that were discovered in the last decode of the 20 th century. These were applied to produce a powerful weapon system for use aboard interstellar spaceships. Such a system became necessary to defend Federation ships from the aggression of the sophisticated warships of the hostile Klingon and Romulan empires.

The key characteristics of the phaser system is its need for very high energy levels that are available on short notice. The basic energy source is the violent interaction of matter and antimatter, which is controlled by means of a catalytic plasma produced from ionized dilithium crystals. Find the matter-antimatter power source on the diagram. The normal result of contact between matter and anti-matter is a violent explosion. However, the catalytic plasma slows the rate at which energy is released, so that use of this energy becomes practical.

The phaser requires energy of several giga-electron volts to be applied within a few picoseconds. Not even the dilithium-based matter-antimatter system can generate such peak levels, and so the energy that it does produce must be stored. The storage system is an outgrowth of the first successful unified field theory. A circulating field, known as an energon ring, can be collapsed by the injection of large amounts of energy from the matter-antimatter power source. The extent of the collapse is determined by the amount of energy injected. Find the matter-antimatter power source on the diagram, and notice the arrows that show the flow of power into the energon storage system.

Mointenance of a collopsed energon ring requires a supply of vector bosons which is synchronized with the period of energon circulation. When the energon ring is allowed to expand, all of the energy is released almost instantaneously, with a maximum release time of 3-5 picoseconds. Because the energy must be taken out of the energon ring within picoseconds, the energon storage system must be able to operate at very high speeds. By making the energon storage system as compact as possible. the time needed for energy to travel between components of the system is minimized. This need for compactness was a major foctor that led to adoption of a toroidal (doughnut-shaped) vessel in which the energon rings circulate. On the diagram, find the energon storage system. You will see the toroidal storage vessel shown with the energon rings circulating inside. Notice that the boson generators are mounted around the outside of the vessel. 


\section{Results}

The number of actions tried was counted as in Experiment 2. Table VII shows the mean number of actions tried by each group, averaged over situations. There is a strong main effect of specific information $(p<.01)$, while the effect of the fantasy context and the interaction failed to reach significance (ps>.1).

\section{Discussion}

These results show that the effectiveness of the device model instructions in the first two experiments was not due to either the motivational interest of the fantasy, nor to the how-it-works information about the system components, nor to the general principles underlying the system. Rather, the critical how-it-works information is the specific items of system topology that relate the controls to the components and to the possible paths of power flow. The stimulation model in Kieras (1984) makes use of exactly this information and is able to infer the procedures for operating devices of this type in a simple and general way.

TABLE VII

Mean Number of Actions Tried While Inferring Procedures

\begin{tabular}{lccr}
\hline & \multicolumn{3}{c}{ Fantasy Condition } \\
\cline { 2 - 4 } \multicolumn{1}{c}{ Specific Information } & No Fantasy & Fantasy & Mean \\
\hline No Specitic Information & 24.3 & 17.7 & 21.0 \\
Specific Information & 6.3 & 6.3 & 6.3 \\
Mean & 12.3 & 10.1 & 11.2 \\
\hline
\end{tabular}

\section{CONCLUSIONS}

The results of these three experiments not only show that device model information can have definite and strong facilitative effects, but also show how it does, and what kind of information is critical for an effective device model for this type of device. The earlier attempts to demonstrate effects may have been inconclusive because the wrong choice of task or model information would produce no effects. Furthermore, the explanation that the task becomes more "meaningful" as a result of having a device model can be replaced with a more specific explanation, that the device model helps because it makes possible specific inferences about what the operating procedures must be. 
Thus, the basic principle advanced here is that, in the context of learning procedures for a device, the useful how-it-works knowledge is the knowledge about the internal workings of the system that allows the user to infer exactly how to operate the device. If this definition of what constitutes a useful device model is adopted, several practical suggestions can be offered for when, and what kind of, device model information should be taught to users of a device:

1. The device model information must support inferences about the exact and specific control actions. Thus, for example, teaching users general principles, metaphors, or analogies will be of little value, since these are unlikely to support such precise inferences (cf. Halasz \& Moran, 1982).

2. The relevant how-it-works knowledge can be very superficial and incomplete, because the user does not need to have a full understanding of the system in order to be able to infer the procedures for operating it.

3. Teaching a device model will not always be of value; it depends on whether the user in the actual task situation both needs to infer the procedures, and also needs the supplied information in order to be able to infer the procedures. If the device is very simple, or the procedure is easily learned and practiced, there in fact may be no need for providing a device model. Also, for some devices, users may be able to easily infer a usable device model without explicit instruction. The ordinary telephone is a good example of a device for which supplying a device model is apparently unnecessary.

4. Learning and using a device model may have its own pitfalls. That is, knowledge of the model may be subject to misunderstandings and distortions, like any other knowledge. Thus if the user is taught a device model, but fails to learn it correctly, incorrect inferences will be made, and performance may not be facilitated at all, or may actually be impaired (see Kieras \& Bovair, 1983; Polson, Kieras, Englebeck, \& Willer, 1983).

\section{REFERENCES}

Alexander, J. H. (1982). Computer text-editing: The development of a cognitive skill. Unpublished doctoral dissertation, Department of Psychology, University of Colorado, Boulder.

Bond, N. C., \& Town, D. M. (1979). Troubleshooting complex equipment in the military services: Research and prospects (Technical Report No. 92). University of Southern Califormia, Behavioral Technology Laboratories, Los Angeles.

Foss, D. J., Smith, P. L., \& Rosson, M. B. (1982, November). The novice at the terminal: Variables affecting understanding and performance. Paper presented at the Psychonomic Society Meeting, Minneapolis. 
Gentner, D., \& Gentner, D. R. (1983). Flowing waters or teeming crowds: Mental models of electricity. In D. Gentner \& A. L. Stevens (Eds.), Mental models. Hillsdale, NJ: Erlbaum.

Gentner, D., \& Stevens, A. L. (Eds.). (1983). Mental models. Hillsdale, NJ: Erlbaum.

Halasz, F. G., \& Moran, T. P. (1982). Analogy considered harmful. In Proceedings of a Conference on Human Factors in Computing Systems. New York: ACM.

Halasz, F. G., \& Moran, T. P. (1983). Mental models and problem solving in using a calculator. In Proceedings of CHI'83 Human Factors in Computing Systems. New York: ACM.

Johnson-Laird, P. N. (1980). Mental models in cognitive science. Cognitive Science, 4, 71-115.

Katona, G. (1940). Organizing and memorizing. New York: Columbia University Press.

Kieras, D. E., \& Bovair, S. (1983). The role of a mental model in learning how to operate a device (Technical Report No. 13, UARZ/DP/TR-83/ONR-13). University of Arizona, Department of Psychology, Tucson.

Kieras, D. E. (1984). A simulation model for procedural inference from a mental model for a simple device (Technical Report No. 15, UARZ/DP/TR-84/ONR-15). University of Arizona, Department of Psychology, Tucson.

Kieras, D. E., \& Polson, P. G. (1982). An outline of a theory of the user complexity of devices and systems (Working Paper No. 1). University of Arizona and University of Colorado, Department of Psychology, Tucson and Boulder.

Kieras, D. E., \& Polson, P. G. (in press). An approach to the formal analysis of user complexity. International Journal of Man-Machine Studies.

Norman, D. A. (1983). Some observations on mental models. In D. Gentner \& A. L. Stevens (Eds.), Mental Models. Hillsdale, NJ: Erlbaum.

Polson, P. G., Kieras, D. E., Engelbeck, G., \& Willer, N. L. (1983, November). Effects of mental models on acquisition of operating procedures. Paper presented at the Psychonomic Society Meeting, San Diego.

Wertheimer, M. (1959). Productive thinking. New York: Harper \& Row.

Williams, M. D., Hollan, J. D., \& Stevens, A. L. (1983). Human reasoning about a simple physical system. In D. Gentner \& A. L. Stevens (Eds.), Mental models. Hillsdale, NJ: Erlbaum. 